\title{
ON ALGEBRAIC DIFFERENTIAL EQUATIONS SATISFIED BY AUTOMORPHIC FUNGTIONS
}

\author{
K. MAHLER \\ (Received 27 January 1969) \\ To Bernhard Hermann Neumann on his 60th birthday \\ Communicated by G. B. Preston
}

In the present note, I investigate the question whether automorphic functions of the Fuchsian type can satisfy algebraic differential equations of the second order. Under rather general restrictions on the corresponding group, it is proved that there is no such differential equation.

\section{1}

Let $R$ and $C$ be the field of real numbers, and of complex numbers, respectively, and let $c \neq 0$ be a constant in $C$.

Denote by $G$ an infinite group of real linear transformations

$$
\left(\begin{array}{ll}
\alpha & \beta \\
\gamma & \delta
\end{array}\right): \omega \rightarrow \frac{\alpha \omega+\beta}{\gamma \omega+\delta} \quad(\alpha, \beta, \gamma, \delta \in R ; \alpha \delta-\beta \gamma=1)
$$

of the upper complex half-plane

$$
H: I(\omega)>0
$$

into itself, and by $f(\omega)$ a non-constant meromorphic function in $H$ which is automorphic with respect to $G$,

$$
f\left(\frac{\alpha \omega+\beta}{\gamma \omega+\delta}\right)=f(\omega) \quad \text { for all }\left(\begin{array}{ll}
\alpha & \beta \\
\gamma & \delta
\end{array}\right) \in G .
$$

We associate with $G$ the set of quotients

$$
S=\left\{\frac{\alpha}{\gamma} \mid\left(\begin{array}{ll}
\alpha & \beta \\
\gamma & \delta
\end{array}\right) \in G\right\},
$$

and we denote by $S^{\prime}$ and $S^{\prime \prime}$ the first and second derived sets of $S$, respectively. Thus $S^{\prime}$ consists of all limit points $\theta$ of $S$, and to each such point $\theta$ there exists an infinite sequence 


$$
\Sigma(\theta)=\left\{\left(\begin{array}{ll}
\alpha_{r} & \beta_{r} \\
\gamma_{r} & \delta_{r}
\end{array}\right)\right\} \quad(r=1,2,3, \ldots)
$$

of elements of $G$ such that

$$
\lim _{r \rightarrow \infty} \alpha_{r} / \gamma_{r}=\theta
$$

Further every element $\Theta$ of $S^{\prime \prime}$ is the limit of an infinite sequence

$$
\left\{\theta_{1}, \theta_{2}, \theta_{3}, \ldots\right\}
$$

of elements of $S^{\prime}$, and to each of these elements $\theta_{s}$ there naturally belongs a set $\Sigma\left(\theta_{s}\right)$.

In order to arrive at a simple result, we impose on the group $G$ the following two restrictions.

$\left(H_{1}\right)$ For each element $\theta$ of $S^{\prime}$, the sequence $\Sigma(\theta)$ can be chosen such that

$$
\lim _{r \rightarrow \infty}\left|\gamma_{r} \delta_{r}\right|=\infty, \quad \lim _{r \rightarrow \infty} \gamma_{r} / \delta_{r}=0
$$

$\left(H_{2}\right) \quad$ The second derived set $S^{\prime \prime}$ contains at least one element.

From $H_{1}$ and $H_{2}$, the following result will be deduced.

THEOREM 1. The five functions

$$
\omega, q(\omega)=e^{c \omega}, f(\omega), f^{\prime}(\omega), f^{\prime \prime}(\omega)
$$

are algebraically independent over $C$.

The proof of this theorem will be indirect, and it will be carried out in a number of separate steps.

\section{2}

We begin with a simple lemma.

Lemma 1. Let $p\left(u_{1}, u_{2}\right)$ be a polynomial in $C\left[u_{1}, u_{2}\right]$ which does not vanish identically, and let $\left\{\theta_{1}, \theta_{2}, \theta_{3}, \ldots\right\}$ be an infinite sequence of distinct elements of $S^{\prime}$ tending to a limit $\Theta$ in $S^{\prime \prime}$. Then

$$
p\left(\theta_{r}, e^{c \theta_{r}}\right) \neq 0
$$

for all sufficiently large suffixes $r$.

PRoOF. If the assertion is false, the regular function $p\left(\omega, e^{c \omega}\right),=g(\omega)$ say, vanishes in an infinite sequence of points converging to $\Theta$, and so $g(\omega)$ vanishes identically. This would mean that $e^{c \omega}$ is an algebraic function of $\omega$, and this is false. 
If $\left(\begin{array}{ll}\alpha & \beta \\ \gamma & \delta\end{array}\right)$ is any element of $G$ such that $\gamma \neq 0$, then

$$
\frac{\alpha \omega+\beta}{\gamma \omega+\beta}=\frac{\alpha}{\gamma}-\frac{1}{\gamma(\gamma \omega+\delta)},
$$

and therefore

$$
q\left(\frac{\alpha \omega+\beta}{\gamma \omega+\delta}\right)=e^{\kappa \alpha / \gamma} \exp \left(\frac{-c}{\gamma(\gamma \omega+\delta)}\right) .
$$

Further, on differentiating twice the identity (1), it follows that

$$
\begin{aligned}
& f^{\prime}\left(\frac{\alpha \omega+\beta}{\gamma \omega+\delta}\right)=f^{\prime}(\omega)(\gamma \omega+\delta)^{2}, \\
& f^{\prime \prime}\left(\frac{\alpha \omega+\beta}{\gamma \omega+\delta}\right)=f^{\prime \prime}(\omega)(\gamma \omega+\delta)^{4}+2 \gamma f^{\prime}(\omega)(\gamma \omega+\delta)^{3} .
\end{aligned}
$$

Since the second formula is rather complicated, it is advantageous to introduce the further function

$$
f^{*}(\omega)=\frac{f^{\prime \prime}(\omega)}{f^{\prime}(\omega)^{2}}
$$

for which, by (6),

$$
f^{*}\left(\frac{\alpha \omega+\beta}{\gamma \omega+\delta}\right)=f^{*}(\omega)+\frac{2 \gamma}{f^{\prime}(\omega)(\gamma \omega+\delta)}
$$

All the four equations (4), (5), (6), and (7) hold identically in $\omega$.

\section{4}

It is evident from the definition of $f^{*}(\omega)$ that Theorem 1 is equivalent to the assertion that the five functions

$$
\omega, q(\omega), \quad f(\omega), f^{\prime}(\omega), f^{*}(\omega)
$$

are algebraically independent over $C$. This assertion will now be proved step by step.

Lemma 2. The two functions $f(\omega)$ and $f^{*}(\omega)$ are algebraically independent over $C$.

Proof. Since, by hypothesis, $f(\omega)$ is not a constant, the two functions can be algebraically dependent over $C$ only if there exists a positive integer $n$ and a set of $n+1$ polynomials $p_{0}(u), p_{1}(u), \ldots, p_{n}(u)$ in $C[u]$ such that $p_{0}(u) \neq 0$ and that 


$$
p_{0}(f(\omega)) f^{*}(\omega)^{n}+p_{1}(f(\omega)) f^{*}(\omega)^{n-1}+\ldots+p_{n}(f(\omega)) \equiv 0
$$

identically in $\omega$. Denote by $\theta$ any point in $S^{\prime}$, and apply to $\omega$ in this identity the transformations $\left(\begin{array}{ll}\alpha_{r} & \beta_{r} \\ \gamma_{r} & \delta_{r}\end{array}\right)$ in $\Sigma(\theta)$. It follows then from $(1)$ and the second formula (6) that the algebraic equation

$$
p_{0}(f(\omega)) x^{n}+p_{1}(f(\omega)) x^{n-1}+\ldots+p_{n}(f(\omega))=0
$$

has infinitely many distinct roots

$$
x=f^{*}(\omega)+\frac{2 \gamma_{r}}{f^{\prime}(\omega)\left(\gamma_{r} \omega+\delta_{r}\right)} \quad(r=1,2,3, \ldots),
$$

which is impossible.

Lemma 3. The four functions $\omega, e^{c \omega}, f(\omega)$, and $f^{*}(\omega)$ are algebraically independent over $C$.

PROOF. If the assertion is false, there exists a polynomial

$$
P\left(u_{1}, u_{2}, u_{3}, u_{4}\right) \neq 0
$$

in $C\left[u_{1}, u_{2}, u_{3}, u_{4}\right]$ such that

$$
P\left(\omega, q(\omega), f(\omega), f^{*}(\omega)\right) \equiv 0
$$

identically in $\omega$. Denote again by $\theta$ any point in $S^{\prime}$, and apply here to $\omega$ the transformations in $\Sigma(\theta)$. It follows then from (1), (4), (5), (6), and (7), that for each suffix $r$,

$$
\begin{aligned}
P\left(\frac{\alpha_{r}}{\gamma_{r}}-\frac{1}{\gamma_{r}\left(\gamma_{r} \omega+\delta_{r}\right)}, e^{c \alpha_{r} / \gamma_{r}} \exp \left(\frac{-c}{\gamma_{r}\left(\gamma_{r} \omega+\delta_{r}\right)}\right),\right. \\
\left.\quad f(\omega), f^{*}(\omega)+\frac{2 \gamma_{r}}{f^{\prime}(\omega)\left(\gamma_{r} \omega+\delta_{r}\right)}\right) \equiv 0
\end{aligned}
$$

identically in $\theta$. Here we allow $r$ to tend to infinity and find in the limit that

$$
P\left(\theta, e^{c \theta}, f(\omega), f^{*}(\omega)\right) \equiv 0,
$$

again identically in $\omega$. Since $P\left(u_{1}, u_{2}, u_{3}, u_{4}\right) \neq 0$, it is, by Lemma 1 , possible to select a limit point $\theta$ in $S^{\prime}$ such that also the polynomial $P\left(\theta, e^{c \theta}, u_{3}, u_{4}\right)$ in the only two indeterminates $u_{3}$ and $u_{4}$ does not vanish identically. The identity (8) implies therefore that $f(\omega)$ and $f^{*}(\omega)$ are algebraically dependent over $C$, contrary to Lemma 2 .

\section{5}

Assume, finally, that Theorem $\mathbf{l}$ is false and that therefore the five functions $\omega, q(\omega), f(\omega), f^{\prime}(\omega)$, and $f^{*}(\omega)$ are algebraically dependent over 
$C$. It follows then from Lemma 3 that there exist a positive integer $N$ and $N+1$ polynomials

where

$$
P_{k}\left(u_{1}, u_{2}, u_{3}, u_{5}\right) \quad(k=0,1, \ldots, N),
$$

such that

$$
P_{0}\left(u_{1}, u_{2}, u_{3}, u_{5}\right) \neq \equiv 0
$$

$$
\sum_{k=1}^{N} P_{k}\left(\omega, q(\omega), f(\omega), f^{*}(\omega)\right) f^{\prime}(\omega)^{N-k} \equiv 0
$$

identically in $\omega$. Again we apply here to $\omega$ all the transformations of a set $\Sigma(\theta)$, and so find that

$$
\begin{aligned}
P_{k}\left(\frac{\alpha_{r}}{\gamma_{r}}-\right. & \frac{1}{\gamma_{r}\left(\gamma_{r} \omega+\delta_{r}\right)}, e^{c \alpha_{r} / \gamma_{r}} \exp \left(\frac{-c}{\gamma_{r}\left(\gamma_{r} \omega+\delta_{r}\right.}\right) \\
& \left.f(\omega), f^{*}(\omega)+\frac{2 \gamma_{r}}{f^{\prime}(\omega)\left(\gamma_{r} \omega+\delta_{r}\right)}\right)\left\{f^{\prime}(\omega)\left(\gamma_{r} \omega+\delta_{r}\right)\right\}^{N-k}=0
\end{aligned}
$$

identically in $\omega$. Divide here by $\left(\gamma_{r} \omega+\delta_{r}\right)^{N}$ and allow $r$ to tend to infinity. Then, in the limit, we find that

$$
P_{0}\left(\theta, e^{c \theta}, f(\omega), f^{*}(\dot{\omega})\right)\left(f^{\prime}(\omega)\right)^{N}=0
$$

identically in $\omega$. However, by what was proved in the last section, $\theta$ can be chosen such that the first factor on the left-hand side does not vanish identically; and the second factor is not identically zero by the hypothesis. This concludes the proof.

\section{6}

From Theorem 1, it follows in particular that the automorphic function $f(\omega)$ does not satisfy any algebraic differential equation of order 2 . On the other hand, under suitable restrictions on the automorphic group $G$, it is well known that $f(\omega)$ may satisfy an algebraic differential equation of order 3 .

An analogous result holds for the function of $q$ defined by

$$
F(q)=f\left(\frac{\log q}{c}\right)
$$

Also $F(q)$ does not satisfy any algebraic differential equation of order 2, but may in certain cases satisfy one of order 3 .

By way of example, let $G$ be identified with the modular group, $f(\omega)$ with the modular function $j(\omega)$ of Weber, and $c$ with the constant $\pi i$. The function $F(q)$ is then given explicitly by 


$$
F(q)=j\left(\frac{\log q}{\pi i}\right)=\frac{\left(1+240 \sum_{k=1}^{\infty} k^{3} q^{2 k}\left(1-q^{2 k}\right)^{-1}\right)^{3}}{q^{2} \prod_{k=1}^{\infty}\left(1-q^{2 k}\right)^{24}},
$$

and while, with respect to $q$, it does not satisfy any algebraic differential equation of the second order, it is a solution of the following differential equation of the third order,

$$
F^{(3)}=\frac{3 q^{2} F^{\prime 2}-4 q F^{\prime} F^{\prime \prime}-F^{\prime 2}}{2 q^{2} F^{\prime}}-F^{\prime 3}\left(\frac{4}{9 F^{2}}+\frac{3}{8\left(F-12^{3}\right)^{2}}-\frac{23}{72 F\left(F-12^{3}\right)}\right) .
$$

It would have some interest to decide whether Theorem 1 remains valid under less restrictive conditions than our hypotheses $\left(H_{1}\right)$ and $\left(H_{2}\right)$. I conjecture that the single condition $\left(H_{2}\right)$ is already sufficient.

Department of Mathematics

Ohio State University

Columbus 